SOMATIC CELL GENETICS

\section{Exploiting Hybrid Cells}

from our Cell Biology Correspondent

THE contents lists of such journals as the Proceedings of the National Academy of Sciences provide a rough and ready, but nonetheless reliable, guide to the burgeoning areas of biology. And on this criterion somatic cell genetics, stemming from Weiss and Green's discovery that manmouse somatic cell hybrids preferentially shed human chromosomes and Watkins and Harris's finding that inactivated Sendai virus acts as a general agent for mediating cell fusion, is quite obviously such an area. The exploitation of these twin discoveries has in the past few years revealed the chromosomal localization of several human, structural genes and has provided human geneticists with an invaluable adjunct to conventional pedigree analysis. For example, both pedigree analyses and somatic cell genetics indicate that the structural gene loci which specify human glucose-6-phosphate dehydrogenase (G6PD) and hypoxanthine: guanine phosphoribosyltransferase (HGPRT) are linked on the human $X$ chromosome.

Such an established gene linkage group is to a geneticist little more than a challenge; having proven two genes are on one chromosome he immediately asks how closely are they linked and how many map units separate them? And to judge from the work of Miller, Cook, MeeraKhan, Shin and Siniscalco (Proc. US Nat. Acad. Sci., 68, 116; 1971), somatic cell hybrids may provide a new method for tackling such questions. They derived six hybrid lines by fusing murine cells, deficient in HGPRT, with human cells with this enzyme and then growing the hybrids in a selective medium in which, to survive, the cells must retain the human HGPRT gene and, therefore, at least part of the human $X$ chromosome. Of course, if the G6PD gene is closely linked to the HGPRT gene on that chromosome one would anticipate that the surviving hybrids would have both these human enzymes. Miller et al. found, however, that in forty-seven of the 105 clones derived from four of their six hybrid lines there was no detectable G6PD activity. The simplest explanation of this unexpected observation is that these two genes are widely separated on the human $X$ chromosome such that breakages in the $X$ chromosome frequently separate them, and thereafter they are selected for independently. There are, of course, alternative and more recondite explanations, but Miller and his colleagues offer convincing arguments against all of them. They seem to have had the good fortune to be studying two weakly linked, widely separated genes but, as they note by treating cells with agents which induce chromosome breakages, it may be possible to establish map distances, not to mention gene orders, between more closely linked

loci by the same approach. Clearly, the exploitation of hybrid for the formal genetic analysis somatic cells is moving from its infancy.

In the same issue of the Proceedings (ibid., 82), Kusano, Long and Green report the selection of a new suite of human-mouse hybrid cells which retain the human gene for adenine phosphoribosyltransferase (APRT) in spite of having lost all the biarmed human chromosomes. These cells were obtained by fusing mouse 3 T6 cells lacking APRT activity with wild type human, diploid fibroblasts and growing the heterokaryons in a medium containing the antibiotic alanosine which inhibits endogenous synthesis of adenylic acid. Only cells retaining human APRT and therefore capable of taking up adenine from their medium survive. Such survivors shed the human biarmed chromo-

somes but retain the human acrocentric chromosomes. They do not contain detectable murine APRT but have the human enzyme, the gene for which must either reside on an acrocentric chromosome or else some translocation or other rearrangement involving the APRT gene must have occurred in all the survivors. By comparing the human chromosomes in those hybrids which have APRT with a line, Kusano et al. have selected from them, which has lost this enzyme, it should prove possible to assign unambiguously the human APRT gene to a particular human, acrocentric chromosome. Further, these hybrids are ideal material for establishing linkages between this gene and other markers and perhaps eventually such linkage groups will be mapped by the methods Miller et al. are developing.

\title{
Controlling Cellular DNA Synthesis
}

IN next Wednesday's Nature New Biology, J. Salas and H. Green report a pioneering set of experiments which may prove to be the first step in the identification and isolation of proteins which act as switches controlling cellular DNA replication. Recently, increasing numbers of teams have begun attempts to elucidate the nature and function of the factors in serum which induce cultures of cells that have reached their saturation density, beyond which there is no appreciable increase in cell number, to undergo further rounds of division. So far, however, they have remarkably little to show for their efforts and that may have been one of the reasons which led Salas and Green to approach the problem of the control of cellular DNA synthesis in an apparently novel way.

Recently Alberts has devised a method for attaching DNA to cellulose columns and has selected, from extracts of bacteria, proteins with an affinity for DNA. Salas and Green have adopted this procedure, immobilizing calf thymus DNA to cellulose, to fractionate the DNA-binding proteins of a line of mouse 3 T 6 cells. The argument is, of course, that proteins which bind to DNA are much more likely to play a part in DNA metabolism than those which fail to bind.

After isolating the DNA-binding proteins from exponentially dividing and stationary cultures of 3 T 6 cells, which had been allowed to incorporate labelled amino-acids, Salas and Green analysed them by polyacrylamide gel electrophoresis. As might be expected, the electrophoretograms revealed a complex pattern of proteins, but eight chief components ( $\mathrm{P} 1-\mathrm{P} 8)$ were discerned, and the amounts of three of these, P1, P2 and P6, seemed to be correlated with the state of growth of the cells. P6 occurred in large amounts in dividing but not stationary cells, and the amount of P1 and P2 was inversely correlated with cell division.

By comparing the amount of labelled amino-acid incorporated into these proteins by cells blocked in the stage of DNA synthesis, by cells moving through DNA synthesis and by cells during the transition from the resting to the dividing state, Salas and Green have clear evidence that the synthesis of P6 correlates with DNA replication. Because $\mathrm{P} 6$ contains tryptophan, as do P1 and P2, the suggestion that any of these proteins are histones can be eliminated. P6 may, however, be some other protein involved in maintaining the structure of chromatin.

The pattern of labelling of $\mathrm{P} 2$ in cells at various stages in the cell cycle is complex and is, until more data are available, not readily interpretable. The pattern of labelling of P1, on the other hand, is quite compatible with the suggestion that it may be a molecule which prevents the onset of DNA synthesis. It is synthesized in large amounts in resting cells but hardly at all in dividing cells. Cells respond to serum starvation by making more $\mathbf{P 1}$, and serum is known to contain a substance necessary for the initiation of DNA synthesis. When cells are artificially held in the stage of DNA synthesis the synthesis of P1 cannot be detected, and, finally, when cells pass from a resting to a dividing state the synthesis of $P 1$ declines to almost nil and this decline probably precedes the replication of DNA and synthesis of P6. Salas and Green have already begun similar experiments with a 3 T 3 line of mouse cells, which is more susceptible to the inhibition of cell division by cell contact, and with cells transformed by oncogenic viruses. They look like uncovering a long and fascinating story which may ultimately reveal several facets of the mechanism of cellular growth control. 